\title{
Retracing the evolution of kinesin-2 deployment from mouse to worm
}

3

4 Augustine Cleetus ${ }^{1, \dagger}$, Georg Merck ${ }^{1, \dagger}$, Zeynep Ökten ${ }^{1, *}$

5 1Physik Department E22, Technische Universität München, Garching, Germany

6 TThese authors contributed equally to this work

$7 \quad$ *Corresponding author. Tel: +49 89289 12885; E-mail: zoekten@ph.tum.de 


\section{Summary}

During the course of evolution, cells have repurposed homologous motor proteins for the transport of an astonishingly diverse set of intracellular cargo. One prominent example of such diversification is the deployment of the respective kinesin-2 homologs in C. elegans (CeOSM-3) and mouse (MmKIF-17). While CeOSM-3 is deployed exclusively inside the cilium as a transport motor, its mouse counterpart behaves as a ciliary cargo. Instead, MmKIF-17 powers many different transport processes in the cytoplasm. Here we turned to functional reconstitution assays to uncover the molecular underpinnings of kinesin-2 deployment. Based on our findings, we propose that cells specified adaptor proteins as strictly conserved 'on- and off-switches', and 'tailored' the motors to fit the respective switches. This is exemplified by the surprising ability of the mouse adaptor to function as a ciliary on-switch for the distantly related CeOSM-3, but not for its own MmKIF-17 motor. Worms, in contrast, retained ciliary on- and off-switches, and can thus deploy CeOSM-3 as a regulatable transport motor inside the cilium. MmKIF-17 escaped this regulatory control by losing its interaction with the ciliary on-switch and has evidently been tailored to fit many different cytoplasmic adaptors over the course of evolution. We could further trace the robustness of an adaptor to serve as a conserved on-switch back to a few strictly conserved residues, which are also present in unicellular organisms. It is therefore conceivable that eukaryotes started to engineer dedicated on- and off-switches early in the evolution and kept their function conserved up to mammals. 


\section{Introduction}

30 Nearly all eukaryotic cells deploy hundreds of different myosins, kinesins, and dyneins to transport an astonishingly diverse set of intracellular cargo on the actin and microtubule cytoskeleton. This already daunting infrastructural problem is further complicated by the fact that a given myosin, kinesin or dynein motor can recognize many different types of cargo molecules and function as versatile transporters in different cells. Such functional plasticity is particularly obvious for the so-called toolbox motors that are common to unicellular as well as to animal cells ${ }^{1}$. The heterotrimeric kinesin-II motor from this toolbox,

for instance, has co-evolved with the near universal ciliary transport machinery to function specifically in Intraflagellar Transport (IFT), and has later been adapted for diverse transport processes in the cytoplasm ${ }^{2,3}$.The kinesin-2 family of motors in fact represents a particularly intriguing case as their deployment differs considerably between species even within the same cellular compartment. The unicellular green algae $C$. reinhardtii for example builds its flagella with the atypically fast heterotrimeric kinesin-II motor ${ }^{4-6}$. The multicellular worm $C$. elegans deploys a second, homodimeric kinesin-2 (CeOSM-3) to build its cilia ${ }^{7,8}$ (Figure 1A). Curiously, while the mouse homolog of the CeOSM-3 motor, the MmKIF-17 kinesin-2, is also found in the cilium, it does not appear to function as an IFT motor ${ }^{9-12}$. Consistent with this notion, specific inhibition of the heterotrimeric kinesin-

47 II completely inhibited the IFT-train movement ${ }^{9}$. Furthermore, MmKIF-17 that lacked its catalytic motor domains still reached the ciliary tip, suggesting that this mammalian

49 kinesin-2 is indeed an inactive cargo, rather than the driver of the IFT-trains (Figure 1B) ${ }^{10,11}$. On the other hand, and in stark contrast to the CeOSM-3, the mammalian 
(Figure $1 \mathrm{~B})^{13}$. The functionally diversified deployment of the homodimeric kinesin-2
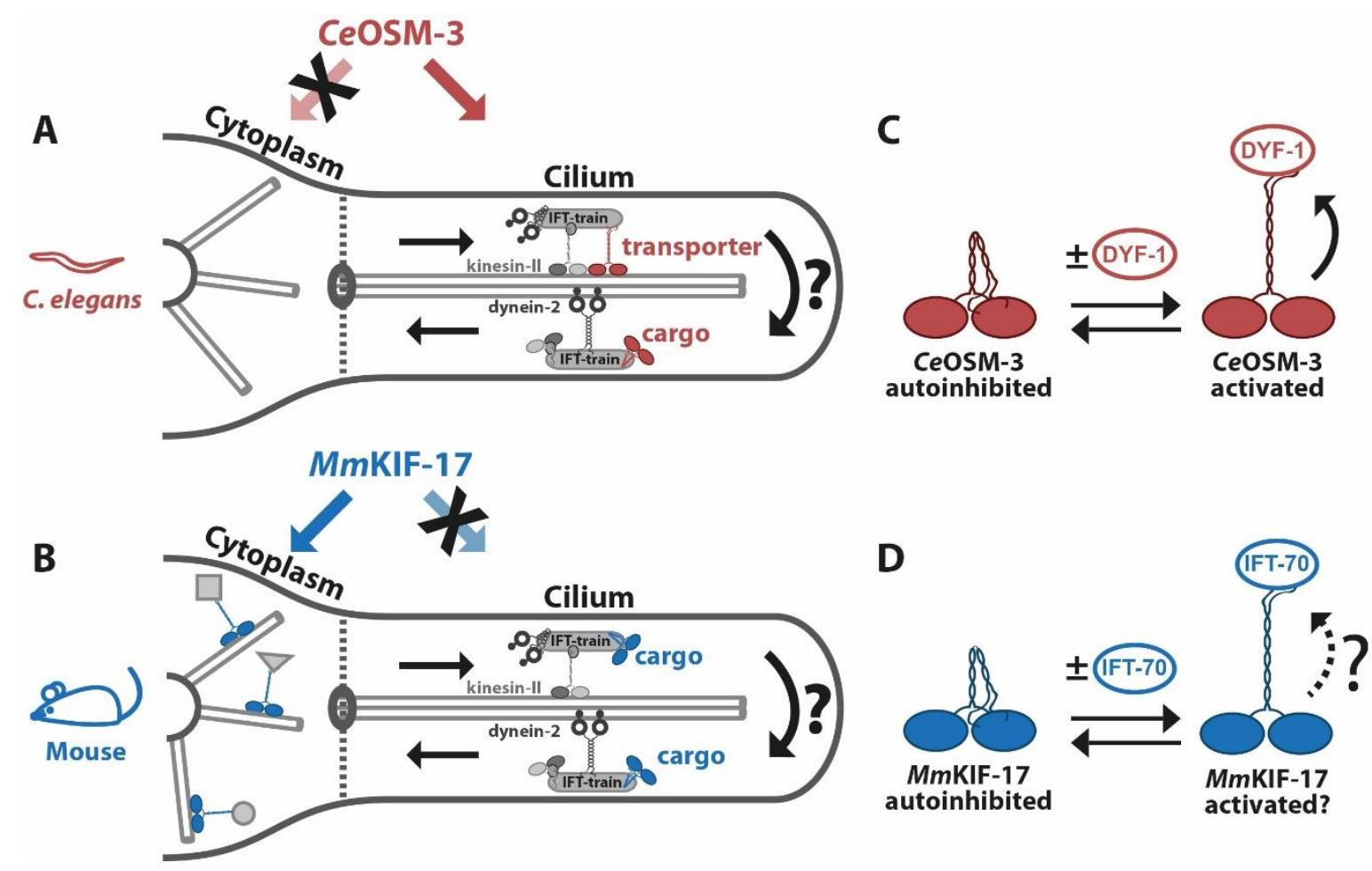

Figure 1: Evolution of the kinesin-2 deployment. (A) To power its IFT-trains towards the ciliary tip, C. elegans deploys a heterotrimeric kinesin-II and a homodimeric CeOSM-3 kinesin-2 motor (the relevant $C$. elegans components are color-coded in red hereafter). The dynein-2 motor is carried as an inactive cargo of the kinesin-2-driven trains. At the ciliary tip, the IFT-train undergoes an enigmatic remodeling process (indicated by curved arrows) that activates the dynein-2 motor for the transport towards the ciliary base and turns the CeOSM-3 motor into an inactive cargo. The CeOSM-3 kinesin-2 is deployed exclusively in the cilium and has never been observed in the cytoplasm (B) Further down the evolution, mouse has apparently evolved a mechanism to turn its homodimeric MmKIF-17 kinesin-2 into a constitutive cargo of IFT-trains inside the cilium (relevant mouse components are color-coded in blue hereafter). Instead, the motor is redirected into the cytoplasm to work as an active transporter of many different cargoes. (C) Illustration of

67 the autoinhibited $\mathrm{CeOSM}-3$ motor. The $\mathrm{C}$-terminal end folds onto the $\mathrm{N}$-terminal head domains to 68 suppress the ATPase activity of the motor via unknown mechanisms. Binding to the CeDYF-1 
adaptor releases the heads and allows the CeOSM-3 motor to move directionally on the microtubules. (D) Whether the adaptor-mediated recruitment and activation mechanism has been conserved up to the respective mouse homologs MmKIF-17 and MmIFT-70 is unknown.

Despite being adapted to diversified transport processes ${ }^{1,3}$, virtually all long-range transport motors share common mechanistic properties, including the CeOSM-3 and MmKIF-17 kinesin-2 homologs. These molecular transporters are processive, meaning that a single motor can take multiple, ATP-dependent 'steps' on its respective filament in vitro $^{14-17}$. This processive stepping, however, is regulated strictly by a common autoinhibitory mechanism. Kinesins can simply 'switch off' their ATPase activity through an inhibitory folding of their C-terminus onto their N-terminal head domains (Figures $1 \mathrm{C}$ and D) $)^{1,18-20}$. Binding of the inhibitory C-terminus to the designated cargo molecule eventually releases the catalytic heads and activates the motor for directional movement (Figures $1 \mathrm{C}$ and D) ${ }^{19,21,22}$. In the vast majority of cases, however, motor recruitment to a specific cargo is mediated by adaptors ${ }^{23-26}$. In the case of CeOSM-3, the conserved CeDYF-1 adaptor protein mediates binding of the motor to its designated cargo, i.e. IFTtrains in vivo and in vitro (Figure $1 \mathrm{C})^{27,28}$. The fact that the mouse MmKIF-17 homolog is no longer deployed as a ciliary transporter thus provokes the question of how the functional relationship between the MmKIF-17 and its corresponding adaptor MmIFT-70 evolved when compared to the CeOSM-3/DYF-1 motor-adaptor complex (Figure 1D). Finally, given the concept of motor activation through adaptor-mediated cargo binding 19,21,22, how can the autoinhibited MmKIF-17 motor bind to the IFT-train but still remain inactive? Here, we address these questions in functional bottom-up reconstitution assays towards a molecular understanding of kinesin-2 deployment and regulation. 


\section{Results and Discussion}

93 Given that MmKIF-17 behaves as an inactive cargo of IFT-trains in vivo (Figure 1B) ${ }^{9-12}$, it is conceivable that the mouse MmIFT-70 adaptor has lost its ability to activate MmKIF17 , or the motor has lost its ability to interact with the adaptor protein for activation, or both (Figures 1D). To distinguish between these possibilities, we used a total internal reflection fluorescence (TIRF) microscope to directly observe the adaptor-regulated transport behavior of single motors on surface-attached microtubules. For our functional bottom-up reconstitution assays, we recombinantly expressed the respective proteins with appropriate tags for fluorescent labeling (Figure S1 and see Supplementary Information for corresponding protein sequences of all constructs used in this study).

The mouse adaptor protein activates the worm but not the mouse kinesin-2

As seen previously with the CeOSM-3 kinesin-2 from C. elegans ${ }^{29}$, its homolog MmKIF-

17 from mouse failed to display any directional movement and occasionally diffused along surface-attached microtubules (Movie S1, left panel). This behavior suggests that the fulllength MmKIF-17 motor construct is autoinhibited in our assays ${ }^{30}$. If so, disengagement of the inhibitory C-terminus either by binding to an artificial cargo or simply by its removal should activate the MmKIF-17 motor. Indeed, surface attachment of the full-length MmKIF-17(1-1038) via its C-terminus as well as the C-terminal truncation MmKIF-17(1747) activated the respective motors to levels that were consistent with previous in vivo 111 studies (Movie S1, right panel, Movie S2, and Figure S2) ${ }^{30-32}$. These results demonstrate 112 that the recombinantly expressed MmKIF-17 construct is fully functional in our 113 reconstitution assays and suggests that the diffusive behavior of the full-length motor 114 results from the C-terminus-mediated autoinhibition ${ }^{30}$. 
115 Next, we asked whether the autoinhibited MmKIF-17 interacts with MmIFT-70, and if so, 116 whether this interaction relieves the autoinhibition of the motor to allow directional

117 transport. To this end, we first assessed if MmKIF-17 motors colocalize efficiently with the

118 MmIFT-70 adaptor protein. In particular, we tested the full-length MmKIF-17 along with 119 the truncated MmKIF-17(1-747) motor that lacked its C-terminus as a control for C120 terminal adaptor binding (Figure 1D). MmKIF-17 with or without its C-terminus failed to 121 display efficient colocalization with the MmIFT-70 protein suggesting that the motor lost 122 its ability to interact with its designated adaptor protein altogether (Figure 2A, first and 123 second lanes). Consistent with this finding, in the presence of the MmIFT-70 adaptor, the 124 MmKIF-17 motor remained autoinhibited in our functional transport assays (Movie S3, top 125 left panel). 


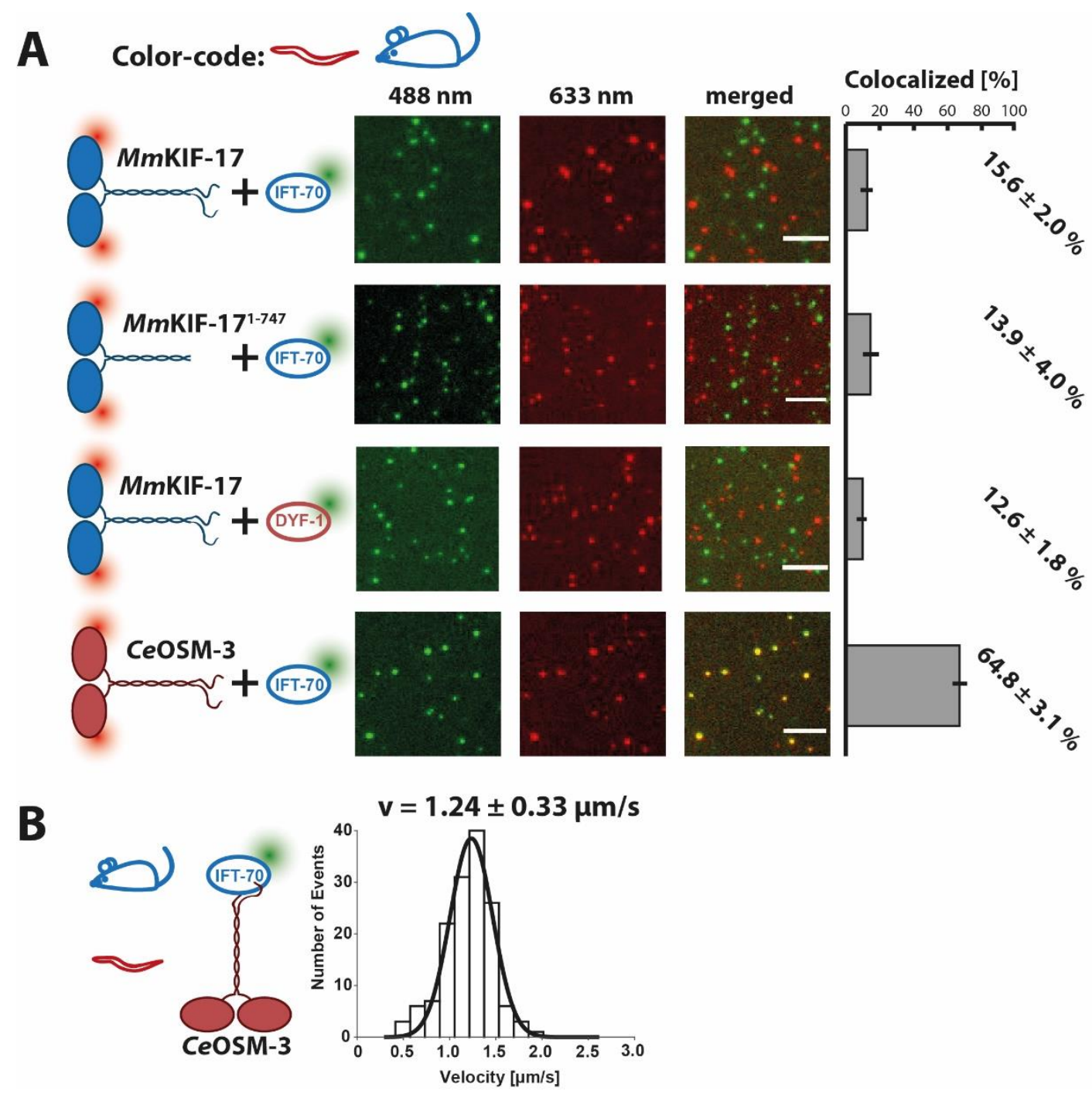

127 Figure 2: Mammalian MmIFT-70 adaptor retained its ability to function as an activator for

128 the kinesin-2 motor. (A) (First and second lanes) The full-length MmKIF-17 ${ }^{\text {SNAP647 }}$ as well as the truncated MmKIF-17(1-747) SNAP647 motors (633 nm channel) failed to efficiently interact with the mouse MmIFT-70 SNAP488 adaptor (488 nm channel) as judged from their colocalization efficiencies

131 ('merged' channel and quantification on the right). (Third lane) The CeDYF-1 ${ }^{\text {SNAP488 }}$ adaptor from

132 C. elegans also failed to interact with the mouse MmKIF-17 motor. (Fourth lane) The CeOSM-

$1333^{\text {SNAP647 }}$ motor not only interacted with the MmIFT-70 SNAP488 adaptor from mouse, (B) but was also

134 activated robustly by MmIFT-70 ${ }^{\text {SNAP488 }}$ (velocity $=1.24 \pm 0.33 \mu \mathrm{m} / \mathrm{s}$; runlength $=2.2 \pm 0.4 \mu \mathrm{m}$, see

135 Movie S3, bottom right panel). Error bars indicate standard deviation (S.D.) from three

136 independent protein preparations with ten images each. Scale bar $=5 \mu \mathrm{m}$. The velocity data was

137 fitted to a Gaussian ( \pm width of distribution) distribution, the runlength data was obtained from a 
single exponential fit ( \pm confidence interval) $(N=145$, from three independent protein preparations).

The failure of the MmIFT-70 adaptor protein to interact with the MmKIF-17 motor immediately provoked the question which of these two proteins evolved to lose this key interaction. To test this, we swapped the adaptors between the species and asked if the CeDYF-1 adaptor from worm activates the mammalian MmKIF-17 motor in vitro, and vice versa. The CeDYF-1 failed to interact with the MmKIF-17 motor as might have been expected from the evolutionary distance between the two organisms (Figure 2A, third lane and Movie S3, top right panel). Strikingly however, the mammalian MmIFT-70 adaptor not only interacted with the distantly related CeOSM-3 motor from worm (Figure 2A, forth lane), but also fully substituted the previously seen function of the worm adaptor CeDYF$1^{27}$, and activated the autoinhibited motor for directional transport (Figure 2B, Movie S3, bottom panels, left vs right). These findings show that the autoinhibited MmKIF-17 motor evolved to lose its ability to interact with the MmIFT-70 adaptor for activation. The MmIFT70 adaptor, in contrast, clearly retained its capability to activate kinesin-2, at least between the $C$. elegans and mouse models. We next asked whether we can unmask the molecular determinants of this remarkable conservation of adaptor function between such distantly related species.

\section{An evolutionary conserved tyrosine motif in DYF-1 is key to kinesin-2 full activation}

The CeDYF-1 adaptor was sufficient to relieve the autoinhibition of CeOSM-3 and activated the motor to a basal level of $\sim 1.2 \mu \mathrm{m} / \mathrm{s}^{27}$ that is faithfully reproduced also by the mammalian MmIFT-70 adaptor protein (Figure 2B). However, when the autoinhibited CeOSM-3 was incorporated into the tetrameric CeDYF-1/DYF-6/OSM-5/OSM-6 complex 
162 via the CeDYF-1 adaptor protein, the motor's speed jumped to $\sim 1.7 \mu \mathrm{m} / \mathrm{s}^{27}$. This full activation of CeOSM-3 indeed corresponds to the in vivo velocity of the motor in the absence of the heterotrimeric kinesin-II motor (Figure $1 \mathrm{~A})^{33}$.

If the CeDYF-1 adaptor is capable of fully activating the CeOSM-3 motor only in the presence of the other three CeOSM-6, CeDYF-6, and CeOSM-5 subunits, it is conceivable that CeDYF-1 must first interact with additional subunit(s) of the complex to fully activate the CeOSM-3 motor. In a previous high-resolution structure, the CeDYF-1 homolog IFT-70 from C. reinhardtii was shown to 'wrap around' a short proline-rich stretch of the CeOSM- 6 homolog IFT-52 ${ }^{34}$. Notably, IFT-52 is one of the most conserved subunits of the IFT-trains ${ }^{35}$ and its absence leads to a complete loss of the cilium in all organisms studied so far $^{36}$. We therefore asked whether this rather unusual interaction of the CeDYF-1 with the universally indispensable CeOSM-6 subunit, as seen with the $C$. reinhardtii homologs, plays a direct role in the full activation of the CeOSM-3 motor. To test this hypothesis, we labeled the CeDYF-1 and the CeOSM-6 subunits with different fluorophores, along with the CeDYF-1 and CeOSM-5 subunits as a control (illustrated in Figures $3 \mathrm{~A}$ and $\mathrm{B}$, left panels). Consistent with previous findings ${ }^{27,29}$, in the absence of any adaptor protein, CeOSM-3 remained autoinhibited and was incapable of directional movement (Movie S4, top left panel), just like the MmKIF-17 motor (Movie S1, left panel). Both, CeDYF-1/OSM-6 and CeDYF-1/OSM-5 adaptors activated the CeOSM-3 motor as judged from the colocalized movement of the respective adaptor subunits (Movie S4, top middle and right panels). However, presence of the OSM-5 subunit failed to fully activate the CeOSM-3, and the motor moved at its basal velocity of $\sim 1.2 \mu \mathrm{m} / \mathrm{s}$ (Figure $3 \mathrm{~A}$ ). The presence of the CeOSM- 6 subunit, on the other hand, was necessary and sufficient to 

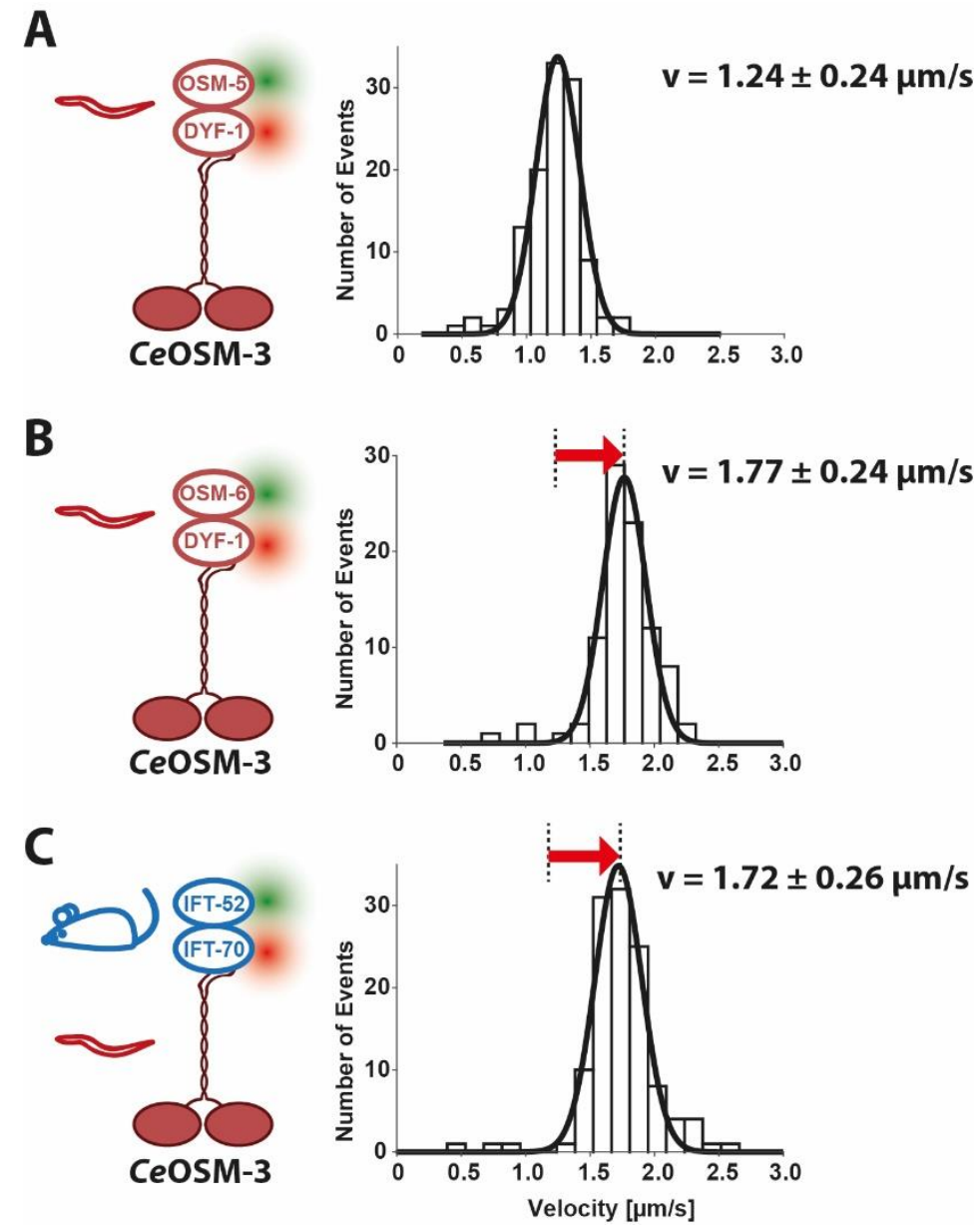

Figure 3: Worm CeDYF-1/OSM-6 and mouse MmIFT-70/IFT-52 subunits are necessary and sufficient for the full activation of the worm CeOSM-3 motor. (A vs B) CeDYF-1 ${ }^{\text {SNAP647 fully }}$ activates the CeOSM-3 motor only in the presence of CeOSM-6 ${ }^{\mathrm{GFP}}(\mathrm{B}$, velocity $=1.77 \pm 0.24 \mu \mathrm{m} / \mathrm{s}$, runlength $=3.9 \pm 0.8 \mu \mathrm{m}, \mathrm{N}=91)$ but not in the presence of the CeOSM-5 ${ }^{\mathrm{GFP}}$ subunit ( $\mathrm{A}$, velocity= $1.24 \pm 0.24 \mu \mathrm{m} / \mathrm{s}$, runlength= $3.2 \pm 0.6 \mu \mathrm{m}, \mathrm{N}=117$ ). (B vs C) Mouse MmIFT-52 ${ }^{\mathrm{GFP}} / \mathrm{IFT}-70^{\mathrm{SNAP} 647}$ adaptor reproduces the full activation of the CeOSM-3 motor as shown in B (velocity $=1.72 \pm 0.26$ $\mu \mathrm{m} / \mathrm{s}$, runlength $=3.0 \pm 0.5 \mu \mathrm{m}, \mathrm{N}=120$ ). Velocity data were fitted to a Gaussian ( \pm width of distribution) distribution (see Table SI for two sample t-tests of the velocities). The runlength data was obtained from a single exponential fit ( \pm confidence interval). Data were obtained from three

197 independent protein preparations each. 
Remarkably, the mammalian MmIFT-70/IFT-52 adaptor faithfully substituted for the CeDYF-1/OSM-6 adaptor function, and fully activated the CeOSM-3 motor from worm (Figure $3 \mathrm{C}$ vs B and Movie S4, bottom panels). These findings suggest that the respective MmIFT-70/IFT-52 and CeDYF-1/OSM-6 adaptors from mouse and worm share common evolutionary conserved signatures that enable the full activation of the CeOSM-3 motor. Here, we focused on a particularly conspicuous repetitive tyrosine motif in the $\mathrm{N}$-terminus

204 of the CeDYF-1 protein that is strictly conserved from unicellular organisms up to 205 mammals (Figure S3) ${ }^{34,37}$. The aromatic rings of these tyrosines in the CeDYF-1 adaptor 206 were seen to tightly stack against the highly conserved proline rings of the CeOSM-6 subunit in the high-resolution structure from the $C$. reinhardtii model ${ }^{34}$. Because this stacking was proposed to stabilize the predominantly hydrophobic interface between the CeDYF-1 and CeOSM-6 proteins ${ }^{34}$, we asked whether interfering with this strictly conserved interface has any functional consequences for the full activation of the CeOSM-3 motor.

212 To test this hypothesis, we simply mutated the repetitive tyrosines into alanines in the 213 CeDYF-1 subunit (Figure 4A). Alanine mutations in the CeDYF-1 adaptor abolished the 214 CeDYF-1/OSM-6-mediated full activation, and the CeOSM-3 moved at a significantly decreased speed that was consistent with the basal activation of the motor (Figures 4B vs C, and Movie S5, left panel) ${ }^{27,29}$. We rescued the full activation of CeOSM-3, however, 217 by mutating the tyrosines into phenylalanines instead of alanines (Figure 4D, and Movie 218 S5, right panel). Phenylalanine is in fact structurally identical to tyrosine, except that it 219 lacks the hydroxyl group $(-\mathrm{OH})$ on the aromatic ring. Based on the capability of 220 phenylalanines to fully substitute the function of wild type tyrosines, we propose that the 
221 stacking between the tyrosines (CeDYF-1) and prolines (CeOSM-6), as seen in the $C$.

222 reinhardtii model ${ }^{34}$, is necessary and sufficient for the full activation of the CeOSM-3

223 motor. The strict conservation of the tyrosine and proline residues that form this

224 hydrophobic interface provides an explanation for the functional compatibility between

225 such distantly related species at the molecular level (Figure S3) ${ }^{34}$. Because these highly

226 conserved repetitive tyrosines are already present in the unicellular organisms such as

227 C. reinhardtii (Figure S3), it is conceivable that the 'on-switch' for the homodimeric

228 kinesin-2 has been specified early in the evolution. 


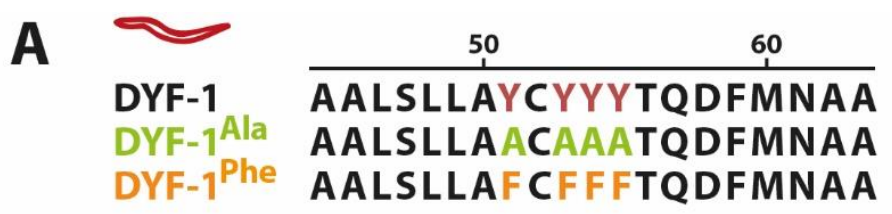

B

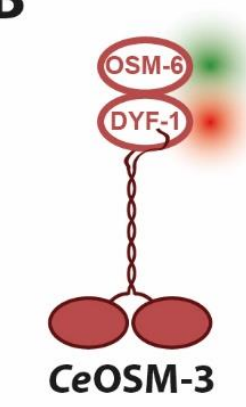

C

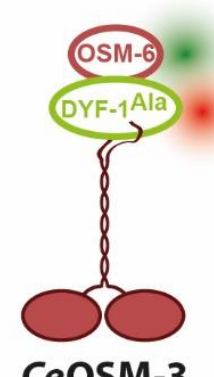

D

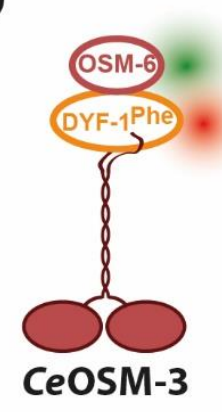

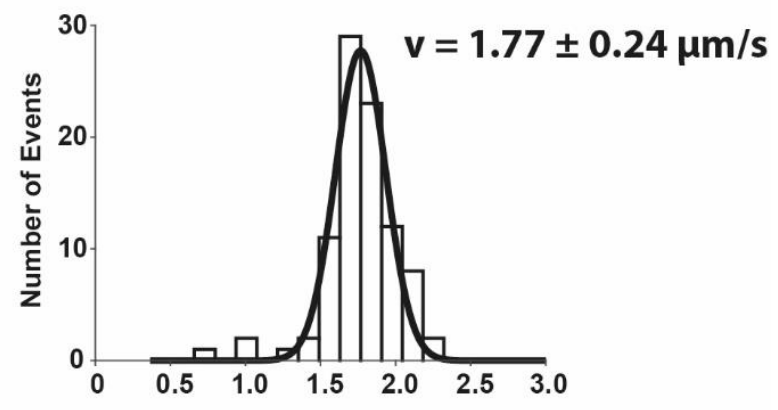
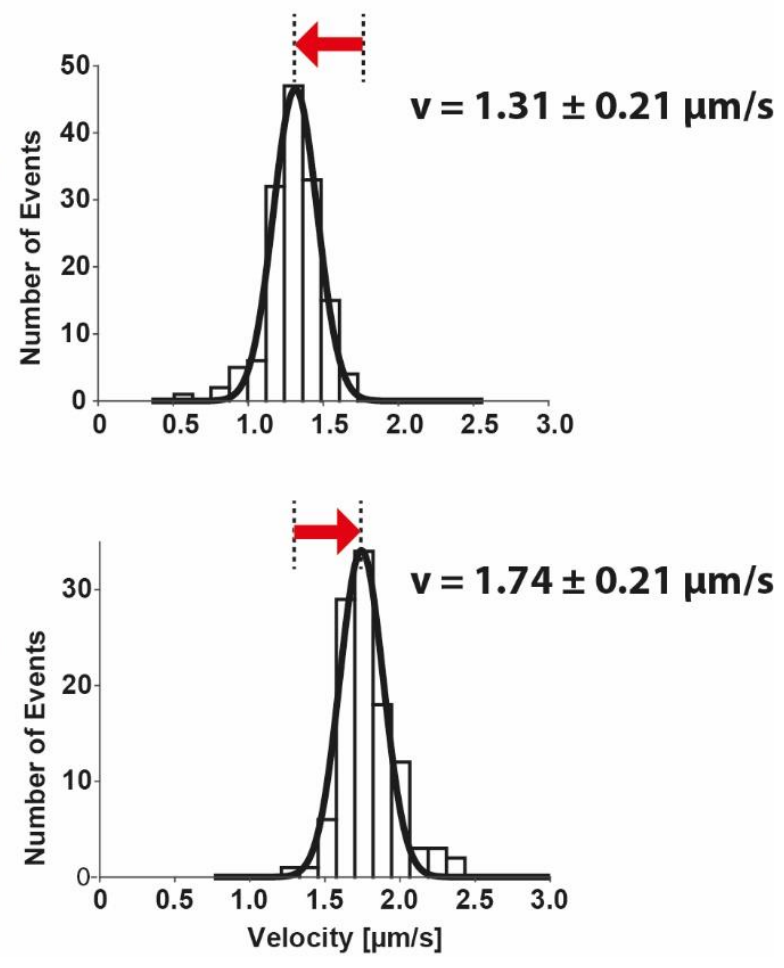

Figure 4: The evolutionary conserved tyrosines in CeDYF-1 are responsible for the full activation of the CeOSM-3 kinesin-2. (A) Sequence alignment of the repetitive tyrosines (see also Figure S3) in the wild type CeDYF-1 (top lane) with the CeDYF-1 ${ }^{\text {Ala }}$ mutant Y51/53/54/55A (green) and CeDYF-1 ${ }^{\text {Phe }}$ mutant Y51/53/54/55F (orange), respectively. (B) The full activation of the CeOSM-3 motor by the wild type CeDYF-1/OSM-6 adaptors replotted from Figure 3B for direct comparison. (C) Mutating the wild type tyrosines into alanines in CeDYF-1 ${ }^{\mathrm{Ala}, \mathrm{SNAP647}}$ prevents the full activation of the CeOSM-3 motor (velocity=1.31 $\pm 0.21 \mu \mathrm{m} / \mathrm{s}$, runlength $=2.4 \pm 0.4 \mu \mathrm{m}, \mathrm{N}=$ 145). (D) The full activation of CeOSM-3 is rescued by replacing the alanines with phenylalanines 
238

239

240

241

242

243

244

245

246

247

248

249

250

251

252

253

254

255

256

257

258

259

in CeDYF- $1^{\text {Phe,SNAP647 }}$ (velocity $=1.74 \pm 0.21 \mu \mathrm{m} / \mathrm{s}$, runlength $=3.3 \pm 0.6 \mu \mathrm{m}, \mathrm{N}=109$ ). Velocity data were fitted to a Gaussian ( \pm width of distribution) distribution (see Table SI for the corresponding two sample t-tests). The runlength was obtained from a single exponential fit ( \pm confidence interval). Data were obtained from three independent protein preparations each.

Taken together, we conclude that the adaptor function is evolutionary conserved as exemplified by the ability of the mouse adaptor to activate the distantly related $C$. elegans motor (Figures 2B and 3C; Movie S4, bottom panels). The mouse motor, on the other hand, evidently underwent a substantial change, as it no longer interacts with its adaptor for activation (Figure 2A, first lane and Movie S3, top left panel).

\section{How is the kinesin-2 motor turned into an inactive cargo?}

The specific, adaptor-mediated recruitment and activation of a motor protein for cargo transport is, however, one side of the coin. The equally important problem of intracellular transport is to also inactivate the motor at the right time and place. This is particularly obvious in the case of the CeOSM-3 motor that transports IFT-trains towards the ciliary tip. On its way out of the cilium, CeOSM-3 is turned into an inactive passenger of the dynein-2-driven IFT-trains (Figure 1A) ${ }^{33,36,38}$. Being an inactive cargo of the IFT-train, however, provokes the question of the molecular mechanism, because adaptor/cargobinding is traditionally equated with the motor activation as detailed above (Figure 1C). In fact, even binding to an artificial cargo, such as a plastic bead, activates autoinhibited kinesins, including the CeOSM-3 motor ${ }^{29,39,40}$. These findings provoke the question of how the CeOSM-3 motor binds to a cargo (i.e. IFT-train) but remains inactive on its way out of the cilium (Figure 1A). 
Robust mechanisms are evidently in place that keep the MmKIF-17 motor as an inactive cargo of the IFT-trains (Figure 1B) ${ }^{9-12}$. Previous in vivo studies already provided clues about the subunits that might be involved in the targeting of the MmKIF-17 kinesin-2 to the IFT-trains. In the absence of ift-56 function, in particular, the motor failed to enter the cilium in vivo ${ }^{12}$. In support, MmKIF-17 motor co-immunoprecipitated with the MmIFT46/IFT-56 subunits from cells ${ }^{12}$. We therefore asked whether we could reconstitute any of these proposed interactions from purified components. Indeed, in the absence of any additional subunits of the IFT-train, MmKIF-17 motor colocalized with the MmIFT-46/IFT56 proteins (Figure 5, first lane). More importantly however, this interaction failed to activate the MmKIF-17 motor in our functional assays (Movie S6, top left panel). Together with previous in vivo findings ${ }^{12}$, our reconstitution studies now provide strong support for the MmIFT-46/IFT-56 subunits to specifically recruit the MmKIF-17 motor to the IFT-trains

272 as an inactive cargo.

Given that the mammalian MmIFT-70/IFT-52 adaptor functioned as an 'on-switch' for the

CeOSM-3 motor from worm (Figure $3 \mathrm{C}$ ), we next asked whether this surprising functional compatibility is also true for the MmIFT-46/IFT-56 adaptor. Indeed, MmIFT-46/IFT-56 interacted with the worm motor (Figure 5, second lane), however, contrary to the MmIFT-

277 70/IFT-52 adaptor that fully activated CeOSM-3 (Figure 3C and Movie S4, bottom panels), binding to MmIFT-46/IFT-56 retained the autoinhibited state of the motor (Movie

279 S6, top right panel). Correspondingly, CeOSM-3 and MmKIF-17 motors interacted with the CeDYF-6/DYF-13 subunits from $C$. elegans which are the homologs of the 281 mammalian MmIFT-46/IFT-56 adaptor (Figure 5, third and fourth lanes). Consistent with the mouse homolog, interaction with the worm CeDYF-6/DYF-13 subunits again failed to 
283 activate the respective motors CeOSM-3 and MmKIF-17 motors (Movie S6, bottom 284 panels).

285 Put simply, MmIFT-46/IFT-56 and CeDYF-6/DYF-13 homologs were functionally 286 indistinguishable for both, the worm CeOSM-3 and mouse MmKIF-17 motors, in our 287 reconstitution assays. The MmIFT-70/IFT-52 and the CeDYF-1/OSM-6 adaptors, in 288 contrast, were indistinguishable only for the CeOSM-3 motor from worm (Figure 3B vs 289 C). In analogy with the MmIFT-70/IFT-52 and CeDYF-1/OSM-6 homologs that functioned 290 as strictly conserved 'on-switch' for the CeOSM-3 motor, we propose that the MmIFT291 46/IFT-56 and CeDYF-6/DYF-13 homologs function as a conserved 'off-switch' due to 292 their ability to keep the respective kinesin-2 motors switched off (Movie 6). 


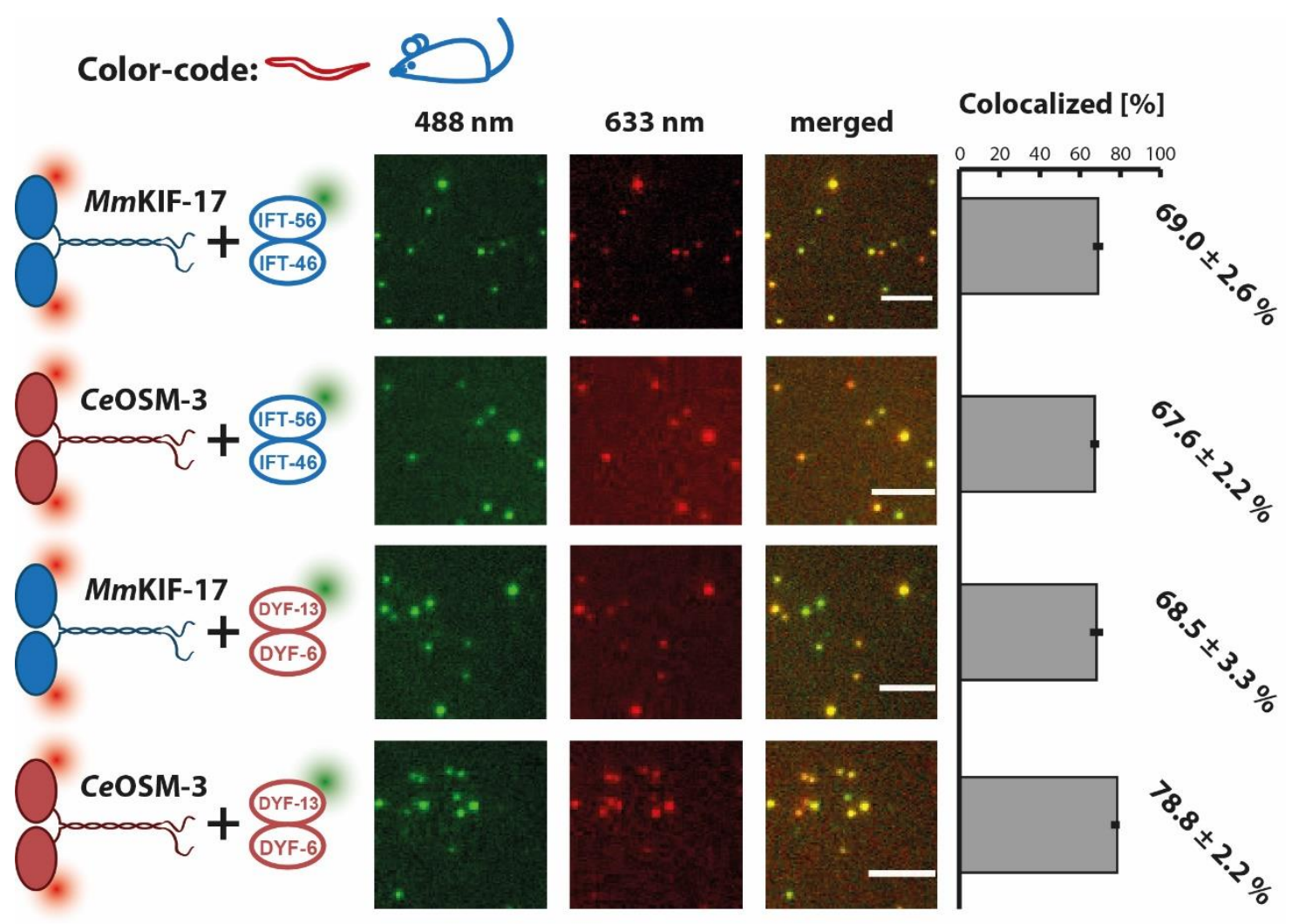

Figure 5: Interaction between the mouse MmIFT-46/IFT-56 and C. elegans CeDYF-6/DYF-13 homologs with the respective kinesin-2 motors is fully conserved. (First and second lanes)

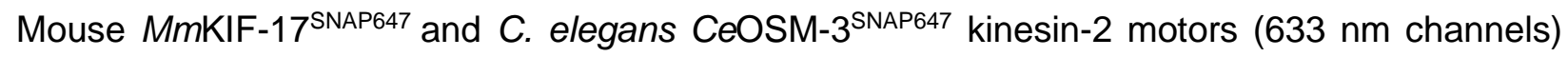
interact with the mouse MmIFT-46/IFT-56 Halo488 adaptor (488 nm channel) as seen in the 'merged' images and the quantification shown on the right. (Third and fourth lanes) Likewise, mouse

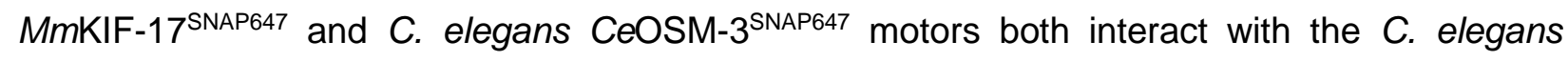
CeDYF-6/DYF-13 ${ }^{\text {SNAP488 }}$ adaptor (488 $\mathrm{nm}$ channel). The quantification on the right shows that the respective interactions between the motors and adaptors are similarly efficient. Error bars indicate standard deviation (S.D.) from three independent experiments with ten images each. Scale bar = $5 \mu \mathrm{m}$.

Together our findings from functional reconstitution studies now provide first molecular clues to explain the long-standing conundrum of kinesin-2 deployment. We propose that the mammalian MmKIF-17 motor has lost its 'on-switch' for ciliary transport during the course of evolution and can no longer be deployed as a regulatable motor for ciliary transport (Figure 6A). The mammalian MmKIF-17 has evidently been 'tuned' to specifically recognize a large set of cytoplasmic adaptors instead, as it transports a 
diverse set of cargo in the cytoplasm (Figure 6A). The opposite is true for the CeOSM-3 motor that has never been observed to transport cytoplasmic cargo, suggesting that worm kinesin-2 has not yet been tuned to 'fit' cytoplasmic adaptors. Inside the cilium, in stark contrast, the CeOSM-3 motor has retained both, an 'on- and an off-switch', and is consequently deployed as a regulatable transport motor (Figure 6B).
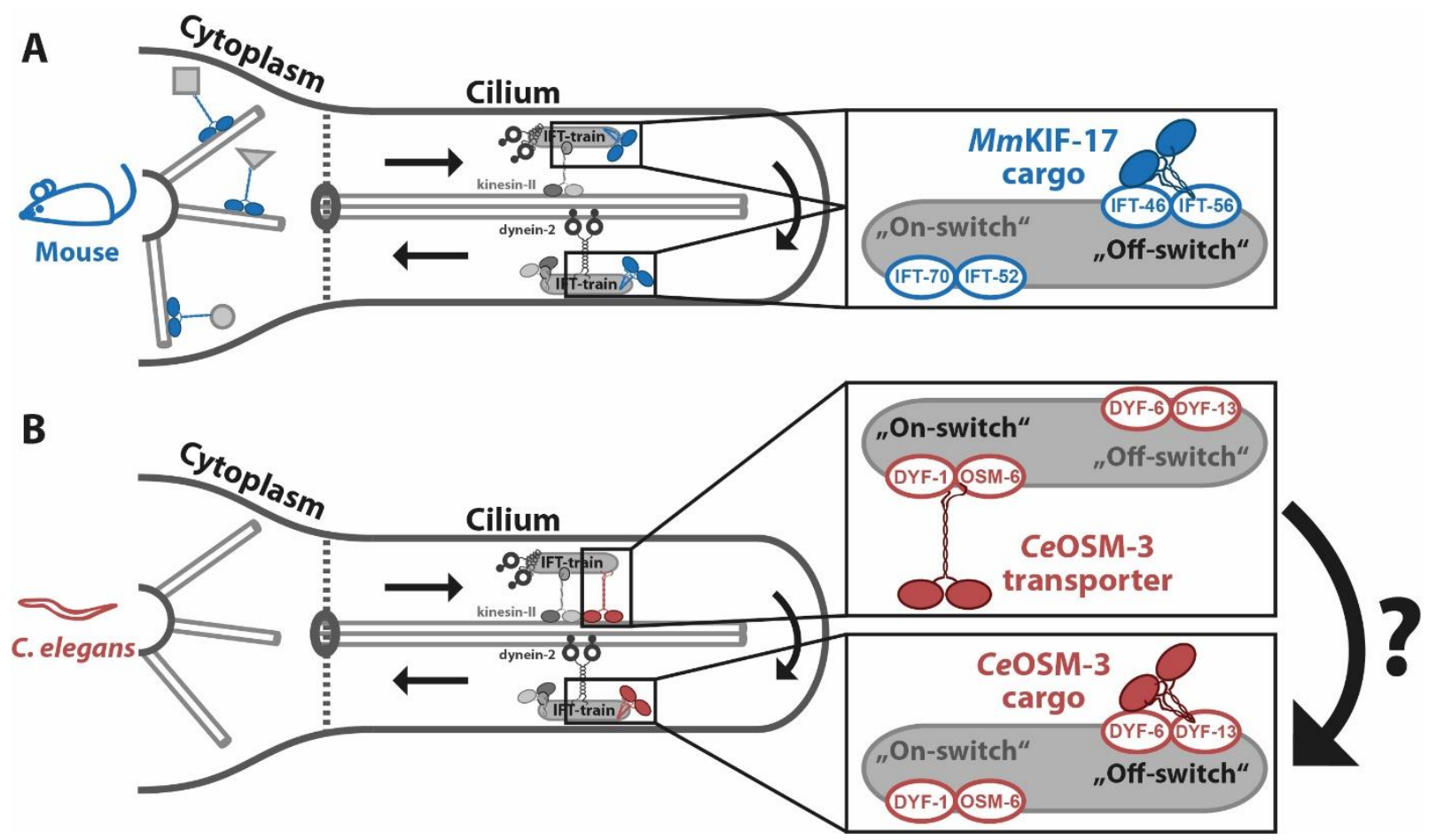

Figure 6: Proposed model of kinesin-2 deployment. (A) The ciliary transport machinery possesses subunits that act as 'on- and off-switches'. This function remains conserved between mouse and worm. In the mice, the MmKIF-17 motor however strayed away during evolution and can no longer utilize the ciliary 'on-switch'. It can still interact with the 'off switch' MmIFT46/IFT56 inside the cilium. Over the course of evolution, MmKIF-17 has been re-adapted to several cytoplasmic 'on-switches' to transport different types of cargo in the cytoplasm via unknown mechanisms. (B) The worm CeOSM-3 motor can utilize both, ciliary on- and off-switches, and can thus be deployed as a regulatable transport motor in the cilium. We propose that the CeOSM3 motor separates from its 'on-switch' and associates with the 'off-switch' to become an inactive cargo of the dynein-2-driven IFT-trains during the remodeling process. Consistent with this proposal, previous in vivo tracking of the CeOSM-3 motor and the CeOSM-6 (subunit of the 'onswitch') showed that the motor turns around without pausing upon arrival at the ciliary tip. Contrary 
to $\mathrm{CeOSM}-3$, the CeOSM-6 subunit is retained significantly longer at the tip suggesting that these two components indeed separate during the remodeling of the IFT-train ${ }^{41}$.

It is, however, important to emphasize that the IFT-train is an exceedingly complex, multisubunit transport machinery ${ }^{36}$. While the MmIFT-46/IFT-56 adaptor is a strong candidate for MmKIF-17 recruitment based on previous in vivo findings ${ }^{12}$, and now our direct interaction studies in functional reconstitution assays (Figure 5 and Movie 6), it is still conceivable that the motor interacts with other subunits of the IFT-train as well. During the assembly and remodeling processes between the ciliary base and the tip, in particular, the MmKIF-17 motor may exchange adaptors on the respective kinesin-2 and dynein-2driven IFT-trains (Figure 6A). Molecular mechanisms of this highly convoluted restructuring of IFT-trains at the ciliary tip, however, remains one of the most enigmatic aspects of ciliary transport to date. Therefore, these questions have to be addressed systematically in bottom-up reconstitution studies to uncover potential interactions between the motor and the IFT subunits and the functional consequences thereof.

In summary, two novel concepts emerged from our functional reconstitution studies. As opposed to the classical view of motor-adaptor interaction that represents the activation step of the autoinhibited motor, we propose that adaptors can have a dual function and serve not only as an 'on-switch', but can also keep the motor switched off. Second, once specified, the adaptor function remains conserved and the motors are 'tailored' for many different adaptors during the course of evolution. The latter is demonstrated particularly well by the MmKIF-17 motor that has been re-adapted to numerous cytoplasmic transport processes over the course of evolution ${ }^{13}$. The next and non-trivial task will be to unmask 
350 the molecular principles of how motors differentiate between many different types of

351 adaptors and how this interaction impacts the activity of the motor.

\section{Data availability}

353 The datasets generated during and/or analyzed during the current study are available 354 from the corresponding author upon request.

\section{Acknowledgements}

356 This work was supported by the European Research Council grant 335623 and by 357 Deutsche Forschungsgemeinschaft grant SFB-863, project ID 111166240 to Z.Ö. We 358 thank Günther Woehlke (Technische Universität München) for fruitful discussions on the 359 project and Felix Müller-Planitz for carefully reading the manuscript (Technische 360 Universität Dresden). We also thank Thi-Hieu Ho and Mohamed Antar Aziz Mohamed 361 (Technische Universität München) for their help in virus generation.

\section{Author contributions}

363 A.C., G.M. and Z.Ö. designed the experiments. A.C. and G.M. performed experiments 364 and analyzed the data. A.C., G.M. and Z.Ö. wrote the manuscript.

\section{Conflict of interest}

366 The authors declare no competing interests. 


\section{References}

3681 Vale, R. D. The molecular motor toolbox for intracellular transport. Cell 112, 467-480, doi:10.1016/s0092-8674(03)00111-9 (2003).

3702 Mitchell, D. R. The evolution of eukaryotic cilia and flagella as motile and sensory organelles. Adv Exp Med Biol 607, 130-140, doi:10.1007/978-0-387-74021-8_11 (2007). 3 Scholey, J. M. Kinesin-2: a family of heterotrimeric and homodimeric motors with diverse 101512-122335 (2013).

4 Sonar, P. et al. Kinesin-2 from C. reinhardtii Is an Atypically Fast and Auto-inhibited Motor that Is Activated by Heterotrimerization for Intraflagellar Transport. Current Biology 30, 11601166.e1165, doi:https://doi.org/10.1016/j.cub.2020.01.046 (2020).

$5 \quad$ Kozminski, K. G., Beech, P. L. \& Rosenbaum, J. L. The Chlamydomonas kinesin-like protein FLA10 is involved in motility associated with the flagellar membrane. The Journal of cell biology 131, 1517-1527, doi:10.1083/jcb.131.6.1517 (1995).

6 Mueller, J., Perrone, C. A., Bower, R., Cole, D. G. \& Porter, M. E. The FLA3 KAP subunit is required for localization of kinesin-2 to the site of flagellar assembly and processive anterograde intraflagellar transport. Molecular biology of the cell 16, 1341-1354, doi:10.1091/mbc.e04-100931 (2005).

7 Signor, D., Wedaman, K. P., Rose, L. S. \& Scholey, J. M. Two Heteromeric Kinesin Complexes in Chemosensory Neurons and Sensory Cilia of Caenorhabditis elegans. Molecular Biology of the Cell 10, 345-360, doi:10.1091/mbc.10.2.345 (1999).

8 Snow, J. J. et al. Two anterograde intraflagellar transport motors cooperate to build sensory cilia on C. elegans neurons. Nat Cell Biol 6, 1109-1113, doi:10.1038/ncb1186 (2004).

9 Engelke, M. F. et al. Acute Inhibition of Heterotrimeric Kinesin-2 Function Reveals Mechanisms of Intraflagellar Transport in Mammalian Cilia. Curr Biol 29, 1137-1148.e1134, doi:10.1016/j.cub.2019.02.043 (2019).

10 Jiang, L. et al. Kinesin family 17 (osmotic avoidance abnormal-3) is dispensable for photoreceptor morphology and function. The FASEB Journal 29, 4866-4880, doi:10.1096/fj.15-275677 (2015). Williams, C. L. et al. Direct evidence for BBSome-associated intraflagellar transport reveals distinct properties of native mammalian cilia. Nat Commun 5, 5813, doi:10.1038/ncomms6813 (2014). Funabashi, T. et al. Ciliary entry of KIF17 is dependent on its binding to the IFT-B complex via IFT46-IFT56 as well as on its nuclear localization signal. Mol Biol Cell 28, 624-633, doi:10.1091/mbc.E16-09-0648 (2017).

14 Endow, S. A. Kinesin motors as molecular machines. BioEssays 25, 1212-1219, doi:10.1002/bies.10358 (2003).

406

407

\section{Howard, J. Vol. 55 (2001).}

6 Svoboda, K., Schmidt, C. F., Schnapp, B. J. \& Block, S. M. Direct observation of kinesin stepping by optical trapping interferometry. Nature 365, 721-727, doi:10.1038/365721a0 (1993).

408 Opin Cell Biol 21, 59-67, doi:10.1016/j.ceb.2008.12.002 (2009). reveals the mechanism of autoinhibition. Science 333, 883-885, doi:10.1126/science.1204824 (2011). 
41219 Verhey, K. J. \& Hammond, J. W. Traffic control: regulation of kinesin motors. Nat Rev Mol Cell Biol 10, 765-777, doi:10.1038/nrm2782 (2009).

20 Adio, S., Reth, J., Bathe, F. \& Woehlke, G. Review: regulation mechanisms of Kinesin-1. J Muscle Res Cell Motil 27, 153-160, doi:10.1007/s10974-005-9054-1 (2006).

21 Blasius, T. L., Cai, D., Jih, G. T., Toret, C. P. \& Verhey, K. J. Two binding partners cooperate to activate the molecular motor Kinesin-1. J Cell Biol 176, 11-17, doi:10.1083/jcb.200605099 (2007).

22 Tafoya, S. \& Bustamante, C. Molecular switch-like regulation in motor proteins. Philos Trans R Soc Lond B Biol Sci 373, doi:10.1098/rstb.2017.0181 (2018).

23 Akhmanova, A. \& Hammer, J. A., 3rd. Linking molecular motors to membrane cargo. Curr Opin Cell Biol 22, 479-487, doi:10.1016/j.ceb.2010.04.008 (2010).

24 Hirokawa, N., Noda, Y., Tanaka, Y. \& Niwa, S. Kinesin superfamily motor proteins and intracellular transport. Nat Rev Mol Cell Biol 10, 682-696, doi:10.1038/nrm2774 (2009).

25 Fu, M. M. \& Holzbaur, E. L. Integrated regulation of motor-driven organelle transport by scaffolding proteins. Trends Cell Biol 24, 564-574, doi:10.1016/j.tcb.2014.05.002 (2014).

26 Cross, J. A. \& Dodding, M. P. Motor-cargo adaptors at the organelle-cytoskeleton interface. Curr Opin Cell Biol 59, 16-23, doi:https://doi.org/10.1016/i.ceb.2019.02.010 (2019).

27 Mohamed, M. A. A., Stepp, W. L. \& Ökten, Z. Reconstitution reveals motor activation for intraflagellar transport. Nature 557, 387-391, doi:10.1038/s41586-018-0105-3 (2018).

28 Ou, G., Blacque, O. E., Snow, J. J., Leroux, M. R. \& Scholey, J. M. Functional coordination of intraflagellar transport motors. Nature 436, 583-587, doi:10.1038/nature03818 (2005).

29 Imanishi, M., Endres, N. F., Gennerich, A. \& Vale, R. D. Autoinhibition regulates the motility of the C. elegans intraflagellar transport motor OSM-3. J Cell Biol 174, 931-937, doi:10.1083/jcb.200605179 (2006).

30 Hammond, J. W., Blasius, T. L., Soppina, V., Cai, D. \& Verhey, K. J. Autoinhibition of the kinesin-2 motor KIF17 via dual intramolecular mechanisms. J Cell Biol 189, 1013-1025, doi:10.1083/jcb.201001057 (2010).

31 Wong, R. W., Setou, M., Teng, J., Takei, Y. \& Hirokawa, N. Overexpression of motor protein KIF17 enhances spatial and working memory in transgenic mice. Proc Natl Acad Sci U S A 99, 1450014505, doi:10.1073/pnas.222371099 (2002).

32 Guillaud, L., Setou, M. \& Hirokawa, N. KIF17 dynamics and regulation of NR2B trafficking in hippocampal neurons. J Neurosci 23, 131-140, doi:10.1523/jneurosci.23-01-00131.2003 (2003).

33 Prevo, B., Mangeol, P., Oswald, F., Scholey, J. M. \& Peterman, E. J. Functional differentiation of cooperating kinesin-2 motors orchestrates cargo import and transport in C. elegans cilia. Nat Cell Biol 17, 1536-1545, doi:10.1038/ncb3263 (2015).

34 Taschner, M., Kotsis, F., Braeuer, P., Kuehn, E. W. \& Lorentzen, E. Crystal structures of IFT70/52 and IFT52/46 provide insight into intraflagellar transport B core complex assembly. Journal of Cell Biology 207, 269-282, doi:10.1083/jcb.201408002 (2014).

35 Brazelton, W. J., Amundsen, C. D., Silflow, C. D. \& Lefebvre, P. A. The bld1 mutation identifies the Chlamydomonas osm-6 homolog as a gene required for flagellar assembly. Curr Biol 11, 15911594, doi:10.1016/s0960-9822(01)00485-7 (2001).

36 Taschner, M. \& Lorentzen, E. The Intraflagellar Transport Machinery. Cold Spring Harb Perspect Biol 8, doi:10.1101/cshperspect.a028092 (2016).

37 Fan, Z. C. et al. Chlamydomonas IFT70/CrDYF-1 is a core component of IFT particle complex B and is required for flagellar assembly. Mol Biol Cell 21, 2696-2706, doi:10.1091/mbc.e10-03-0191 (2010).

38 Prevo, B., Scholey, J. M. \& Peterman, E. J. G. Intraflagellar transport: mechanisms of motor action, cooperation, and cargo delivery. Febs j 284, 2905-2931, doi:10.1111/febs.14068 (2017). 
39 Coy, D. L., Hancock, W. O., Wagenbach, M. \& Howard, J. Kinesin's tail domain is an inhibitory regulator of the motor domain. Nat Cell Biol 1, 288-292, doi:10.1038/13001 (1999).

$46140 \quad$ Friedman, D. S. \& Vale, R. D. Single-molecule analysis of kinesin motility reveals regulation by the Turnarounds of Intraflagellar Transport at the C. elegans Ciliary Tip. Cell Rep 25, 1701-1707.e1702, doi:10.1016/j.celrep.2018.10.050 (2018).

466

467 


\section{Materials and methods}

\section{DNA constructs, design and virus generation}

All DNA sequences were based on published information from NCBI (https://www.ncbi.nlm.nih.gov/) and were commercially synthesized by GenScript (GenScript Biotech, Piscataway Township, NJ, USA). All DNA constructs were cloned into the pFastBac-1 vector according to the manufacturer's instructions (Thermo Fischer Scientific, Waltham, MA, USA). To facilitate protein purification, constructs were FLAGor His-tagged, respectively. For fluorescent labeling, SNAP-, Halo-, or GFP-tags were used as detailed in the supporting information. Recombinant bacmid generation and virus amplification were done according to the manufacturer's constructions (Thermo Fischer Scientific, Waltham, MA, USA).

\section{Protein expression, purification, and fluorescent labeling}

All proteins were expressed following the manufacturer's instructions (Thermo Fischer Scientific, Waltham, MA, USA) using the Baculovirus Expression System in Sf9 insect cells (Spodoptera frugiperda). All proteins were purified with either a FLAG-tag or His-tag affinity purification via a $\mathrm{N}$ - or C-terminal FLAG (DYKDDDDK) or 6xHis-tag. The following protocol refers to a $50 \mathrm{ml}$ cell suspension culture with a concentration of approx. $2 \times 10^{6}$ cells/ml cultured in Sf-900 ${ }^{\mathrm{TM}}$ II SFM medium supplemented with $10 \%$ (v/v) FBS and 0.5 $\%(w / v)$ Gentamicin. Cell suspension cultures were infected for single protein expression or co-expressions with $2-8 \%(\mathrm{v} / \mathrm{v})$ appropriate virus suspension and incubated in flasks for approx. $60 \mathrm{~h}$ at $28{ }^{\circ} \mathrm{C}$ and $110 \mathrm{rpm}$. For FLAG-tag purification cells were centrifuged for 10 mins at $2,500 \mathrm{~g}$ at $4^{\circ} \mathrm{C}$ and lysed in $2 \mathrm{ml} \mathrm{FLAG} \mathrm{lysis} \mathrm{buffer}(50 \mathrm{mM}$ Pipes $(\mathrm{pH}=$ 6.9), $300 \mathrm{mM}$ Potassium acetate, $1 \mathrm{mM} \mathrm{MgCl}_{2}, 1 \mathrm{mM}$ DTT, $0.1 \mathrm{mM} \mathrm{ATP}, 0.5$ \% Triton X100, $10 \%$ (v/v) Glycerine and Complete Protease Inhibitor Cocktail (Roche, Basel, CH) and centrifuged for 10 mins at $4^{\circ} \mathrm{C}$ and $65,000 \mathrm{~g}$. The supernatant was subsequently incubated with $50 \mu \mathrm{l}$ of ANTI-FLAG MS Affinity Agarose gel (Sigma-Aldrich, St. Louis, $\mathrm{MO}$, USA) for 90 mins at $4{ }^{\circ} \mathrm{C}$ on rolling incubator. Beads were consequently washed three times with $1 \mathrm{ml}$ FLAG wash buffer I (80 mM Pipes $(\mathrm{pH}=6.9), 500 \mathrm{mM}$ Potassium acetate, $1 \mathrm{mM} \mathrm{MgCl}$, $1 \mathrm{mM}$ DTT, $0.1 \mathrm{mM}$ ATP, $0.1 \%$ Tween-20, $1 \mathrm{mM} \mathrm{EGTA,} 10 \%$ (v/v) Glycerine) and three times with $1 \mathrm{ml}$ FLAG wash buffer II (80 mM Pipes (pH = 6.9), 200 mM Potassium acetate, $1 \mathrm{mM} \mathrm{MgCl} 2,1 \mathrm{mM}$ DTT, $0.1 \mathrm{mM}$ ATP, $0.1 \%$ Tween-20, $1 \mathrm{mM}$ EGTA, $10 \%$ (v/v) Glycerine). For fluorescent labelling, the beads were incubated for 40 mins at $4{ }^{\circ} \mathrm{C}$ on a rolling incubator with $200 \mu \mathrm{FLAG}$ wash buffer II and either $1 \mathrm{mM}$ HaloTag ${ }^{\circledR}$ or SNAP surface ${ }^{\circledR}$ Alexa Fluor dyes according to the tag of the protein and desired wavelength of the dye. The beads were subsequently washed another three times with $1 \mathrm{ml}$ FLAG wash buffer II and eluted in $100 \mu \mathrm{l} \mathrm{FLAG} \mathrm{elution} \mathrm{buffer} \mathrm{(FLAG} \mathrm{wash} \mathrm{buffer}$ II with $0.5 \mathrm{mg} / \mathrm{ml}$ Flag peptides (Sigma-Aldrich, St. Louis, MO, USA).

For the His-tag purification, the cell pellet was lysed with $2 \mathrm{ml}$ lysis buffer (FLAG lysis buffer, $20 \mathrm{mM}$ imidazole, $\mathrm{pH}$ 8) and the supernatant was incubated with $80 \mu \mathrm{l}$ pre-washed Ni-NTA coated Sepharose beads (Ni-NTA agarose, (Qiagen, Hilden) for 90 mins at $4^{\circ} \mathrm{C}$ on a rolling incubator. Subsequently, the beads were washed four times with $1 \mathrm{ml}$ wash buffer (FLAG wash buffer I, $40 \mathrm{mM}$ imidazole, $\mathrm{pH}$ 8). For fluorescent labelling, the beads were incubated for 40 mins at $4{ }^{\circ} \mathrm{C}$ on a rolling incubator with $200 \mu$ wash buffer and either 1 mM HaloTag ${ }^{\circledR}$ or SNAP surface ${ }^{\circledR}$ Alexa Fluor dyes according to the tag of the 
protein and desired wavelength. The beads were subsequently washed three times with $1 \mathrm{ml}$ wash buffer and eluted in $100 \mu \mathrm{l}$ of the elution buffer (FLAG wash buffer II, $500 \mathrm{mM}$ imidazole, $\mathrm{pH}$ 7). Purity, yield, and concentration of the purified proteins were analysed using SDS-PAGE.

\section{Multiple motor filament gliding assay}

The gliding assays was performed as described before ${ }^{1}$. Briefly, a flow chamber $(\mathrm{V}=\sim 5$ $\mu \mathrm{l}$ ) was created by fusing a Parafilm between a slide and a cover slip. $6 \mu$ l of motor protein were flown into the chamber and incubated for 2 mins to allow binding of the motor tails to the glass surface. Unbound motors were washed out with $30 \mu$ BRB80 BSA (80 mM Pipes ( $\mathrm{pH}=6.9$ ), $2 \mathrm{mM} \mathrm{MgCl}$, $1 \mathrm{mM}$ EGTA,1mg/ml BSA, $5 \mathrm{mM}$ DTT). Fluorescently labeled microtubules were diluted in $50 \mu$ Motility buffer $(80 \mathrm{mM}$ Pipes $(\mathrm{pH}=6.9), 2 \mathrm{mM}$ $\mathrm{MgCl}_{2}, 1 \mathrm{mM}$ EGTA, $5 \mathrm{mM}$ DTT, 2 mM ATP, $0.18 \mathrm{mg} / \mathrm{ml}$ glucose-oxidase (G2133, SigmaAldrich, St. Louis, MO, USA), $0.06 \mathrm{mg} / \mathrm{ml}$ catalase (C3155, Sigma-Aldrich, St. Louis, MO, USA) and $0.4 \%$ glucose) and were flown into the chamber. The movement of the filaments was tracked with an objective-type Leica DMi8 TIRF microscope (Leica microsystems, Wetzlar, Germany) equipped with a plan objective lens (100x, N.A. 1.47 Oil) and an Andor iXon Ultra EMCCD camera (Andor Technology, Belfast, UK). The velocities were analysed with the microscope software AF 6000 (Leica microsystems, Wetzlar, Germany).

\section{Colocalization assays}

Purified proteins were labeled with appropriate dyes as described above and were mixed in equimolar concentration and incubated over night at $4^{\circ} \mathrm{C}$ in a rolling incubator. The respective protein mixtures were pipetted directly onto the glass slide and covered with a cover slip. Images were captured using the TIRF microscope described above. The colocalization of the labelled proteins was analysed using a custom written MATLAB (MathWorks Inc., Natick, MA, USA) routine as described previously².

\section{Single-molecule transport assays}

Purified and fluorescently labelled proteins were mixed in equimolar concentrations and incubated overnight at $4{ }^{\circ} \mathrm{C}$ in a rolling incubator as described above. Biotinylated and fluorescently labelled microtubule filaments were attached to the glass surface of a flow chamber via biotin-streptavidin, as described previously². For single molecule imaging, proteins were diluted in the motility buffer ( $80 \mathrm{mM}$ Pipes $(\mathrm{pH}=6.9), 2 \mathrm{mM} \mathrm{MgCl}, 1 \mathrm{mM}$ EGTA, 5 mM DTT, 2 mM ATP, 0.18 mg/ml glucose-oxidase (G2133, Sigma-Aldrich, St. Louis, MO, USA), $0.06 \mathrm{mg} / \mathrm{ml}$ catalase (C3155, Sigma-Aldrich, St. Louis, MO, USA) and $0.4 \%$ glucose) and flushed into the flow chamber. Fluorescently labelled proteins were excited with the appropriate wavelength and tracked using the TIRF microscope as described above. The runlength and velocity data were analysed using a custom-written MATLAB (MathWorks Inc., Natick, MA, USA) routine as described previously². Briefly, data points were selected automatically according to their relative brightness to the background in each frame. Consecutive runs were considered processive with a minimum runlength of $1 \mu \mathrm{m}$ and data points fit to a truncated $\left(\mathrm{x}_{0}=1 \mu \mathrm{m}\right)$ single exponential distribution. Velocity runs were considered as part of distance over time data points of at 
least six frames that resulted in a linear fit with $r^{2}$-values $>95 \%$. Data points for the velocity were subsequently fit to a Gaussian distribution.

For colocalized runs, proteins were simultaneously tracked in both channels ( $488 \mathrm{~nm}$ and $633 \mathrm{~nm}$ ) and analysed in a custom-written MATLAB (MathWorks Inc., Natick, MA, USA) routine as described previously ${ }^{3}$. Briefly, colocalized movements were assigned using a penalty score system for both channels. The penalty score resulted from the mean distances of tracked positions (pixels, factor 1/3) and the difference in the starting time (frames, factor 1). Runs were adjusted to the length of the shorter one to account for possible bleaching. Runs were considered colocalized with a score of $<10$ and their determined parameters were used in subsequent analysis as the average of both runs. Consecutive runs were considered processive with a minimum runlength of $2 \mu \mathrm{m}$ and data points fit to a truncated $\left(x_{0}=2 \mu \mathrm{m}\right)$ single exponential distribution. The cut off was increased to $2 \mu \mathrm{m}$, because the algorithm for the colocalization is more efficient for longer runs.

\section{References}

1. Brunnbauer M, Mueller-Planitz F, Kösem S, Ho T. H., Dombi R., Christof J., Gebhardt M., Rief M., Ökten Z. (2010). Regulation of a heterodimeric kinesin-2 through an unprocessive motor domain that is turned processive by its partner. Proc Natl Acad Sci 107(23):10460-10465

2. Stepp WL, Merck G, Mueller-Planitz F, Ökten Z. (2017). Kinesin-2 motors adapt their stepping behavior for processive transport on axonemes and microtubules. EMBO Rep. 18:1947-1956.

3. Sonar P, Youyen W, Cleetus A, et al. (2020). Kinesin-2 from C. reinhardtii Is an Atypically Fast and Auto-inhibited Motor that Is Activated by Heterotrimerization for Intraflagellar Transport. Curr Biol. 30(6): 1160-1166 